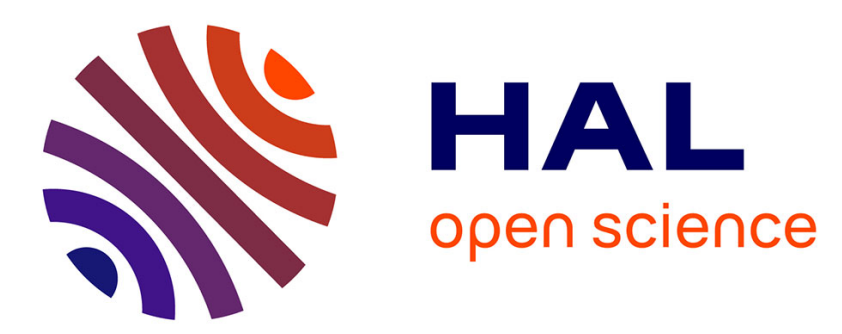

\title{
An integrated model for strategic supply chain design: Formulation and ABC-based solution approach
}

\author{
Linda Zhang, Carman Lee, Shuzhu Zhang
}

\section{To cite this version:}

Linda Zhang, Carman Lee, Shuzhu Zhang. An integrated model for strategic supply chain design: Formulation and ABC-based solution approach. Expert Systems with Applications, 2016, 52, pp.3949. 10.1016/j.eswa.2015.12.035 . hal-01744868

\section{HAL Id: hal-01744868 \\ https://hal.science/hal-01744868}

Submitted on 23 Nov 2018

HAL is a multi-disciplinary open access archive for the deposit and dissemination of scientific research documents, whether they are published or not. The documents may come from teaching and research institutions in France or abroad, or from public or private research centers.
L'archive ouverte pluridisciplinaire HAL, est destinée au dépôt et à la diffusion de documents scientifiques de niveau recherche, publiés ou non, émanant des établissements d'enseignement et de recherche français ou étrangers, des laboratoires publics ou privés. 


\title{
An integrated model for strategic supply chain design: Formulation and ABC- based solution approach
}

\author{
Linda L. Zhang ${ }^{\mathrm{a}}$, Carman Lee ${ }^{\mathrm{b}}$, Shuzhu Zhang ${ }^{\mathrm{b}}$ \\ a Department of Management, IESEG School of Management (LEM-CNRS), 3, rue de la \\ Digue, 59000 Lille, France \\ b Department of Industrial and Systems Engineering, The Hong Kong Polytechnic \\ University, Hong Kong, China
}

\begin{abstract}
This study develops a mixed integer nonlinear programming (MINLP) model to design supply chains. In view of the limitations of many available strategic supply chain design models, this model involves three major supply chain stages, including procurement, production, and distribution, and their interactions; it takes into account bill of materials constraints for modeling complex supply chain inter-relationships. In addition, in accordance with the fact that companies nowadays develop product families, our model addresses multi-product supply chain design to respond to diverse customer requirements. Recognizing their importance, this study identifies and formulates constraints related to facility pairwise relationships and supplier priority along with the classical constraints from the available literature. To efficiently solve such a highly constrained, large scale MINLP model, we develop an approach based on an artificial bee colony $(\mathrm{ABC})$ algorithm. Bicycle design and production is used to demonstrate the potential of the MINLP model for designing supply chains and the performance of the ABC-based solution approach in solving the model. The proposed model and solution approach can be considered as two fundamental components of an expert system in the broad sense. Thus, this study is expected to stimulate more future research on the development of practical expert systems for designing supply chains.
\end{abstract}

\section{Introduction}

A supply chain involves multiple facilities (i.e., supply chain members), such as raw material and component suppliers, final product producers, distribution centers. Based on customer requirements, supply chain members collaboratively design, produce, and deliver products while attempting to achieve the optimal performance of the cohort of the chain (Safaei, Moattar Husseini, \& Farahani, 2010; Zhang, You, Jiao, \& Helo, 2009). As today's business competition is among supply chains, instead of individual firms, it is deemed important to design effective supply chains, which can help sustain competitive advantages for all chain members. This is well evidenced by the numerous articles reported for designing supply chains in the recent two decades (Gebennini, Gamberini, \& Manzini, 2009; Ivanov, 2010).

As pointed out in (Gebennini et al., 2009; Ivanov, 2010; Sabri \& Beamon, 2000; Simchi-Levi, Kaminsky, \& Simchi-Levi, 2004; Thanh, Bostel, \& Peton, 2008), the supply chain design's central decisions

* Corresponding author. Tel.: +33 750600235 .

E-mail addresses: 1.zhang@ieseg.fr (L.L. Zhang), ckm.lee@polyu.edu.hk (C. Lee), shuzhu.zhang@connect.polyu.hk (S. Zhang). include supplier selection, facility location and capacities, customer demand allocation, raw material, component and product flows, which are at the strategic level. To cope with these decisions, researchers have proposed a myriad of valuable strategic supply chain design models. Most of these models treat each stage (or at most two) of the chain as a separate system, e.g., only procurement or only production or the integration of production-distribution; a few studies have addressed an integrated supply chain design from material procurement to production to product delivery (Sabri \& Beamon, 2000; Li, Hendry, \& Teunter, 2009). However, as revealed in industrial projects (Melo, Nickel, \& Saldanha-da-Gama, 2005), companies wish the simultaneous consideration of all the three important stages in their supply chain design. This indicates that designing a supply chain by considering multistage and their interactions yields realistic solutions. In a recent review article based on the analysis of 33 survey-based studies, van der Vaart and van Donk (2008) point out that integrated supply chain design leads to higher supply chain performance. In view of the importance of considering multistage in supply chain design and the relative lack of models, in this study, we simultaneously consider procurement, production and distribution in the strategic supply chain design.

A bill of materials (BOM) is a very important product document and describes in detail product's constituent elements and 
their relationships. BOMs provide key information for coordinating activities between material procurement and production in a supply chain and are, thus, related to many complex supply chain inter-relationships (Yan, Yu, \& Cheng, 2003). Researchers highlight that BOMs should be exploited to coordinate the behavior of suppliers with the production and distribution activities and should be considered as constraints in the strategic supply chain design (Arntzen, Brown, Harrison, \& Trafton, 1995; Cohen \& Lee, 1989; Melo, Nickel, \& Saldanha-da-Gama, 2009). However, due to the difficulties in formulating BOM-related constraints in a mathematical model, there is a lack of models with the inclusion of BOM constraints (Vidal \& Goetschalckx, 1997; Thanh, Bostel, \& Peton, 2008; Melo et al., 2009). In this study, we consider BOMs in the development of the integrated model for strategic supply chain design and formulate BOM-related constraints.

Among the available supply chain design models, some consider one product (Li et al., 2009; Osman \& Demirli, 2010, to name but three); some involve multiple products (Das, 2011; Safaei et al., 2010; Thanh, Bostel, \& Peton, 2008; Yan et al., 2003). Designing supply chains for multiple products is more practical. The reason for this is that in practice, companies develop families of related products (so called product families) to fulfill diverse individualized customer requirements. Due to the similarities among customized products in a family, a supply chain is normally utilized to design and produce one product family (Huang, Zhang, \& Liang, 2005). In this study, we consider multiple products in developing the integrated, strategic supply chain design model.

To summarize, this study focuses on the strategic design of supply chains for multiple products by considering procurement, production, distribution and their interactions and BOM-related constraints. A mixed integer non-linear programming (MINLP) model is developed. In order to solve the MINLP model, swarm intelligence is employed in this study. Swarm intelligence is a new category of the meta-heuristics, which is inspired by the collective intelligence of insect colonies or animal societies in their operational behavior (Bonabeau \& Dorigo, 1999). One of the typical examples of intelligent swarms is the bee colony, which demonstrates an amazing intelligence when foraging for food sources. In this study, we, thus, employ a bee colony inspired algorithm, named artificial bee colony (ABC) algorithm. The $A B C$ algorithm is originally introduced by Karaboga (2005) and becomes a popular choice for solving constrained optimization problems in comparison to the evolutionary computation thanks to its simple yet robust framework and implementation simplicity. The $A B C$ algorithm is efficient in both exploration and exploitation of the search space because of its unique design of multiple roles and phases (Horng, 2011; Karaboga, Gorkemli, Ozturk, \& Karaboga, 2014). In view of the advantage of the $A B C$ algorithm for solving optimization problems, we develop an approach based on the $A B C$ algorithm to solve the proposed MINLP model. The effectiveness and efficiency of the ABC-based solution approach is demonstrated through a comparison with other approaches, including LINDO (a commercial solver) and a genetic algorithm (GA)-based approach.

The rest of the paper is organized as follows. We formulate the MINLP integrated, strategic supply chain design model in Section 2 by identifying diverse constraints. This is followed by the proposed ABC-based solving approach in Section 3. Bicycle design and production is used to demonstrate designing supply chains using the MINLP model in Section 4. Also provided in this section is the comparison between the proposed $\mathrm{ABC}$-based solution approach and LINDO, the GA approach. We conclude this paper in Section 5 by pointing out the limitations and identifying potential avenues for future research.

\section{Model formulation}

\subsection{Problem context and decisions}

A set of products is to be designed, manufactured, and distributed by a set of facilities, including suppliers, production plants, and distribution centers (DCs), which will form a supply chain. A production plant is responsible for the overall design and production activities, whilst suppliers are expected to be involved in design and production of intermediate components. Each facility has limited capacity in fulfilling its tasks. Together, they complete the product design, production, and distribution according to customer requirements. Consistent with the current global manufacturing practice, while the set of production plants belong to one company, the suppliers and DCs may not belong to the same company (Chung, Lau, Choy, Ho, \& Tse, 2010; Kim \& Kim, 2008). In addition, the company makes the decision on the plants, suppliers, and DCs to be included in the supply chain. As indicated by Anussornnitisarn, Nof, \& Etzion, 2005; Dominiquez and Lashkari, 2004; Moon, Seo, Yun, \& Gen, 2006, the above context where supply chains are formed is not uncommon in practice, especially when involving internationally operating companies.

In this study, the general decisions considered are related to the selection of supply chain facilities and the allocation of loading (e.g., component quantities) to these facilities. The specific decisions deal with: (i) the selection of specific suppliers, production plants, and DCs, (ii) the design tasks assigned to suppliers and plants, (iii) the amount of components and products to be produced and shipped among suppliers, plant, DCs, and customers, (iv) global capacity coordination for the involved suppliers and plants, and (v) distribution planning that determines optimal distribution channel and quantity. Taking into account these decision factors, we formulate the below MINLP model.

\subsection{The MINLP model}

Involving multiple products and multiple stages, the supply chain design problem requires a systematic approach to account for product-related characteristics, supply and demand matching, facility loading, and both inter- and intra-facility transactions. The supply chain designed should be cost-effective so that the total cost associated with design, production, holding inventory, and logistics can be reduced to a minimum level. In light of the above issues, the MINLP model attempts to minimize the total cost of a supply chain, subject to the capacities of suppliers, plants, and DCs, throughput constraints, and customer demand requirements as well. The model is formulated based on the following assumptions.

\section{Assumptions:}

(1) Each plant is able to produce any arbitrary product mix;

(2) Product fulfillment within a plant is divided into a finite set of sub-tasks, each being carried out by one or more than one supplier;

(3) If a supplier undertakes a task of designing a component, it is also responsible for producing the component;

(4) Each plant purchases components from multiple suppliers and each supplier serves several plants;

(5) Each DC is opened for any arbitrary product mix;

(6) DCs deliver all received products to customers (Note: Like many available supply chain design models, drop shipping is not considered in this study. Thus, DCs receive products and deliver them to customers.);

(7) According to geographical locations, customers are grouped into different zones, forming customer zones (CZs); 
(8) The design and production capacities of suppliers and plants are known;

(9) The throughputs of plants and DCs are known;

(10) The transportation costs from facilities to facilities are known.

In accordance with the above decisions and assumptions, we define a list of notation, including indices, input parameters, and decision variables (see Appendix A). While most of the definitions, e.g., unit production cost, setup cost, are easily understandable, two input parameters: design capacity and service level are explained below. In line with the uncertainties of development capabilities of a facility, design capacity is the probability that a plant (or supplier) can undertake the design task (Wang \& Lin, 2006). It assumes a real value in $(0,1]$. A service level is measured as the percentage of orders that can be delivered by a supply chain facility (i.e., a supplier, a plant, or a DC) to its downstream facility (Simchi-Levi, Kaminsky, \& Simchi-Levi, 2009). It also assumes a real value in $(0,1]$.

The objective function is to minimize the total cost, $C$, of the entire supply chain during a time period (\$/period) as follows:

$$
\begin{aligned}
\operatorname{Min} C= & {\left[\sum_{r j} U_{r j}\left(\eta_{r j} / \delta_{r j}\right)+\sum_{r j} U_{r j} f_{r j}^{S}+\sum_{r j} U_{r j} p_{r j}^{S} Z_{r j}+\sum_{r j k} U_{r j} c_{r j k}^{S P} R_{r j k}\right] } \\
& +\left[\sum_{i k} V_{i k}\left(\eta_{i k} / \delta_{i k}\right)+\sum_{i k} V_{i k} f_{i k}^{P}+\sum_{i k} V_{i k} p_{i k}^{P} X_{i k}+\sum_{i k l} V_{i k} c_{i k l}^{P D} Q_{i k l}\right] \\
& +\left[\sum_{i l} W_{i l} f_{i l}^{D}+\sum_{i l} W_{i l} p_{i l}^{D} Y_{i l}+\sum_{i l m} W_{i l} c_{i l m}^{D Z} P_{i l m}\right]
\end{aligned}
$$

Subject to:

$$
\begin{aligned}
& \sum_{k} R_{r j k} \leq \Phi_{r j}^{S}, \forall r, j \\
& \sum_{k} R_{r j k} \leq Z_{r j}, \forall r, j \\
& \sum_{j}\left(R_{r j k} \cdot \alpha_{j}\right) \geq \lambda_{r i} X_{i k}, \forall r, i, k \\
& \sum_{l} Q_{i k l} \leq \Phi_{i k}^{P}, \forall i, k \\
& \sum_{i l} Q_{i k l} \leq \Phi_{k}^{P}, \forall k \\
& \sum_{i}\left(Q_{i k l} \cdot \beta_{k}\right) \geq Y_{i l}, \forall k \\
& Y_{i l}=\sum_{m} P_{i l m}, \forall i, l \\
& \sum_{l}\left(P_{i l m} \gamma_{l} T_{i l m}\right) \geq D_{i m}, \forall i, m \\
& \sum_{i} Y_{i l} \leq \Phi_{l m}^{D}, \forall l \\
& \sum_{l} T_{i l m} \geq 1, \forall i, m
\end{aligned}
$$$$
X_{i k}, Y_{i l}, Z_{r j}, P_{i l m}, Q_{i k l}, R_{r j k} \geq 0, \forall i, j, k, l, m
$$$$
U_{r j}, V_{i k}, W_{i l}, T_{i l m}=0 \text { or } 1, \forall i, k, l, m
$$$$
\sum_{j} U_{r j} Z_{r j}-\lambda_{r i} \sum_{k} V_{i k} X_{i k} \geq 0, \quad \text { foralli }
$$

$V_{i k}-C_{r i}\left(U_{r m}+U_{r n}+\cdots+U_{r p}\right) \leq 0$

$W_{i l}-A_{i}\left(V_{i x}+V_{i y}+\cdots+V_{i z}\right) \leq 0$

$U_{r m} \geq U_{r n}$, for $m, n \in J$

The total cost in the above objective function includes both fixed and variable costs. It contains cost factors pertaining to three stages in a supply chain: the procurement, production, and delivery stages. In the procurement stage, the cost consists of component design, production set-up, component purchasing, and transportation costs. Among them, design cost is derived from the supplier's capacity to design a specific component. In the production stage, the cost consists of product design, production set-up, and production ramp up costs, and transportation cost from plants to DCs as well. In the delivery stage, the cost consists of set-up and inventory holding costs of DCs and transportation cost from DCs to CZs.

The quantity of any component that can be shipped from one supplier to plants is limited to the supplier's production capacity, as indicated in Constraint 1 . Constraint 2 ensures the material balance of a supplier, that is, the quantity of a component shipped from a supplier is equal to the total quantity provided by the supplier. Constraint 3 guarantees that the demand for component $j$ of product $i$ from plant $k$ is always met. Constraint 4 is to reinforce the rule: the quantity of a product shipped from a plant cannot be larger than the plant's production capacity. Similarly, Constraint 5 is to ensure that the maximum quantity of mixed products provided by a plant does not exceed the plant's total capacity. To meet the demand of a product at a DC, Constraint 6 is formulated. A DC can always supply the right amount of a product to its CZs (i.e., matching supply to demand), as indicated in Constraint 7. Constraint 8 ensures that the demand of each $C Z$ is satisfied. Constraint 9 limits the total quantity of products that a DC can receive to be no larger than its maximum throughput. Constraint 10 specifies that for any product, each $\mathrm{CZ}$ should be served by at least one DC. Constraint 11 ensures non-negativity of quantities shipped in different stages. Constraint 12 determines the binary variables. Component quantity requirement in accordance with products' BOMs are met in Constraint 13. Constraints 14 and 15 model the pairwise relationship among facilities. Constraint 16 ensures supplier priority.

To sum up, in the above constraint formulation, we consider the classical constraints (e.g., Constraints 1, 2, and 3) appearing in the available supply chain models. Complementing the existing studies, we identify and formulate new constraints associated with products' BOMs (Constraint 13), facility pairwise relationships (Constraints 14 and 15), and supplier priority (Constraint 16). These constraints along with their reasoning and importance are elaborated below.

\subsubsection{BOM constraints}

BOMs contain information about product components, such as raw material, parts and assemblies, their relationships, and their quantities for one unit of the final products. Each component is usually associated with multiple supply chain facilities, be they raw materials (or parts) suppliers, final product production plants, or DCs. BOMs, thus, provide a foundation for selecting suppliers, determining plants, and allocating production quantities in designing supply chains. They are commonly represented graphically (as shown in Fig. 1) or tabularly. Such representation formats do not allow the easy formulation of mathematical expressions. As a consequence, most reported supply chain models only consider the quantity relationships among product components while ignoring 


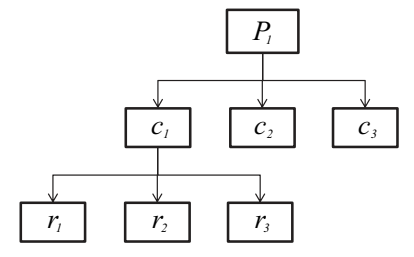

Fig. 1. The BOM structure of product $P_{1}$.

the interactive impact between final products and product components. In this study, we take into account not only the quantity relationships among components but also these between product components and final products, which give rise to interconnections among supply chain facilities, in particular component suppliers and final product producers.

Product $P_{1}$ in Fig. 1 is used to explain the quantity relationships between final products and product components. $P_{1}$ has three immediate child components, including $c_{1}$ (an assembly), $c_{2}$ (a part) and $c_{3}$ (a part). $c_{1}$ is formed by three parts: $r_{1}, r_{2}$, and $r_{3}$. The aggregated quantities of these components are modeled as $n_{r_{1} \rightarrow P_{1}}$, $n_{r_{2} \rightarrow P_{1}}, n_{r_{3} \rightarrow P_{1}}, n_{c_{1} \rightarrow P_{1}}, n_{c_{2} \rightarrow P_{1}}$ and $n_{c_{3} \rightarrow P_{1}}$. Take $n_{r_{1} \rightarrow P_{1}}$ as an example. It indicates that for each unit of $P_{1}$ to be produced in any plant, $n_{r_{1} \rightarrow P_{1}}$ units of $r_{1}$ must be delivered by one or more suppliers. In the light of the above relationships, we formulate Constraint 13 to model the interconnections among supply chain facilities.

\subsubsection{Facility pairwise constraints}

In most available supply chain models, it is commonly assumed that all suppliers/plants serve all plants/DCs (e.g., Li et al., 2009). That is, each supply chain facility is connected with all immediate downstream (or upstream) facilities, indicating a full connectivity, as shown in Fig 2(a). (Note, the connection between two facilities at two adjacent levels of a supply chain is referred to as a facility pairwise relationship.) Although supply chain models formulated based on the full connectivity are easy to solve, they are not practical. This is because this assumption is often not realized in practice. In practice, due to the geographic and economical issues, a supply chain facility normally serves a part of, but not all, its downstream facilities, and receives products (or components) from a subset of its upstream facilities. In other words, each facility is connected with a subset of downstream (or upstream) facilities, indicating a partial connectivity in Fig. 2(b).

Let $S, P$, and $D$ denote the sets of suppliers, plants, and DCs, respectively; $\left\langle p_{k}: s_{j}\right\rangle$ models that plant $p_{k}$ is served by supplier $s_{j}$ for one or more components. Accordingly, the facility pairwise relationship in Fig. 2(b) can be represented as six pairwise tuples: $\left\langle d_{1}\right.$ : $\left.p_{1}\right\rangle,\left\langle d_{2}: p_{1}, p_{2}\right\rangle,\left\langle d_{3}: p_{2}, p_{3}\right\rangle,\left\langle p_{1}: s_{1}\right\rangle,\left\langle p_{2}: s_{1}, s_{2}\right\rangle$ and $\left\langle p_{3}: s_{2}, s_{3}\right\rangle$. Each facility pairwise relationship entails a logical rule. For examples, If plant $p_{j}$ is opened for one or more products, at least one a

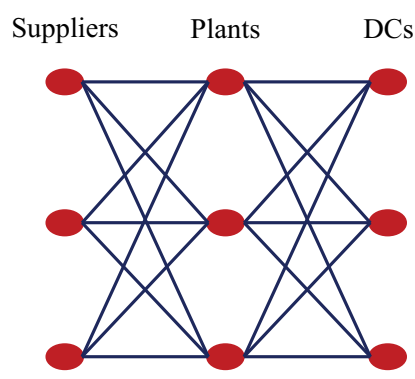

b

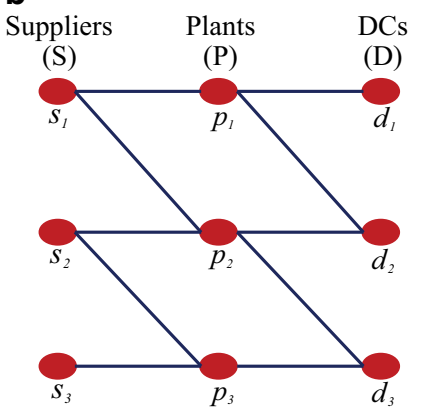

Fig. 2. Pairwise relationship among supply chain facilities. of the suppliers $s_{m}, s_{n}, \ldots s_{p}, m, n, p \in J$ should be opened; if DC $d_{j}$ is opened for one or more products, at least one of the plants $p_{x}, p_{y}, \ldots p_{z}, x, y, z \in K$ should be opened. In this study, we formulate Constraints 14 and 15 to capture these pairwise relationships by following the above logic. While Constraint 14 models the pairwise relationships between a plant and its suppliers, Constraint 15 captures these between a DC and the plants that can potentially serve it.

\subsubsection{Supplier priority constraints}

In practice, companies often take into account preference considerations when selecting suppliers. Preference considerations associate suppliers with different priorities. The implication is that given same or similar conditions (e.g., prices, delivery lead times), a plant may prefer supplier A, instead of supplier B. Suppose that both suppliers $s_{1}$ and $s_{2}$ can produce a same component for plant $p_{1}$. In this regard, either $s_{1}$, or $s_{2}$, or both can be chosen as suppliers for meeting the demand of $p_{1}$. Such general relationships among $p_{1}, s_{1}$, and $s_{2}$ can be represented by three inequalities: $-s_{1}+p_{1} \geq 0,-s_{2}+p_{1} \geq 0$, and $s_{1}+s_{2}-p_{1} \geq 0$. Representing supplier selection in general, these inequalities, however, overlook the preference considerations (i.e., supplier priority).

Suppose $p_{1}$ prefers $s_{1}$ to $s_{2}$. This preference can be modeled as $s_{1}-s_{2} \geq 0$. This inequality ensures that $s_{1}$ always has a higher priority than $s_{2}$. In line with this representation, we formulate Constraint 16 to model facilities' preference considerations when deciding on upstream facilities.

Incorporating such priority constraints in a model can ease problem solving as well. For example, suppose that six suppliers are able to provide same components at similar prices, delivery lead times, and quality. Three of them are required to fulfill a specific demand. Without considering supplier priority, there are $C_{6}^{3}=60$ possible combinations. If supplier priority (in this case, supplier 1 is preferred to supplier 3 , and supplier 4 is preferred to supplier 2) is considered, only 10 possible combinations deserve further investigation, including suppliers 1,4 and 5 , suppliers 1,4 and 6 , suppliers 1,5 , and 6 , suppliers 4,5 and 6 , suppliers 1,3 , and 4, suppliers 1,3 and 5, suppliers 1,3 and 6 , suppliers 4, 2 and 1 , suppliers 4, 2 and 5, and suppliers 4, 2 and 6.

\section{ABC-based solution approach}

The $A B C$ algorithm is a typical example of the swarm intelligence. It simulates the intelligent foraging behavior of honey bees. In a bee hive, there are three types of bees: scout bees, employed bees, and onlooker bees. These bees interact with one another and the environment for finding the best food source around the bee hive. The process of the $A B C$ algorithm consists of four distinct phases, including (1) initialization phase, (2) employed bee phase, (3) onlooker bee phase, and (4) scout bee phase, as shown in Fig. 3.

Involving different types of bees, each phase fulfills certain function. In the initialization phase, the scout bees are sent out to find the initial food sources around the bee hive at random. Subsequently, in the second phrase, the employed bees begin to exploit the food sources. During the course of exploiting the current food sources, the employed bees also try to explore better food sources which are nearby the current ones. Upon finding them, the employed bees replace the previous food sources with these new ones. This is termed as greedy selection in the second phase. During the third phase, the onlooker bees in the hive receive the food sources information from the employed bees and determine probabilistically to follow the employed bees to exploit and explore the food sources. Such determination is proportional to the richness of the food sources. Food sources are gradually consumed and finally exhausted. The exhausted food sources would be abandoned and 


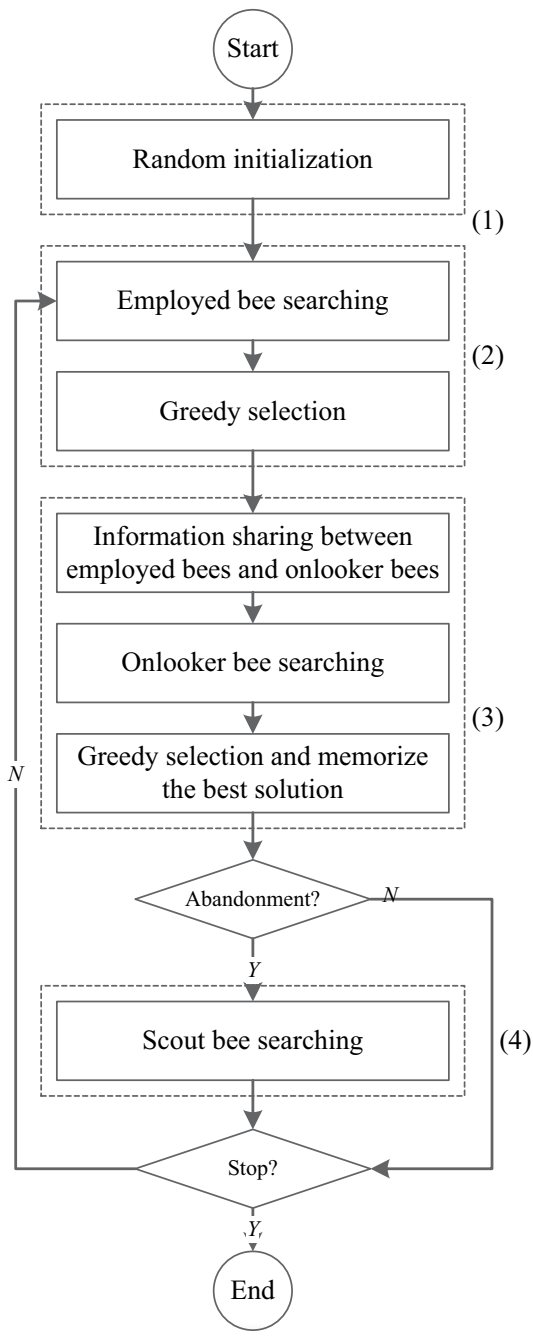

Fig. 3. The process of the $A B C$ algorithm.

replaced by other food sources found by the scout bees in the last phase.

\subsection{Solution scheme}

In the proposed MINLP model, the number of parameters, decision variables and constraints is very large. This is especially true when the supply chain network is complicated. When the network design problem has multiple stages and each echelon involves multiple parties, representing solutions using matrix scheme in the traditional way is not able to model the problem (Yan, Yu, \& Cheng, 2003). Thus, in this study, a hybrid encoding system consisting of a binary-based encoding mechanism and a prioritybased encoding mechanism is proposed to represent the solution scheme. In accordance with the two encoding mechanisms, a solution, thus, has two sections. The binary-based encoding mechanism is employed to denote the open/close status of facilities in the supply chain network, while the priority-based encoding mechanism is employed to indicate the product transportation at each stage (Altiparmak, Gen, Lin, \& Karaoglan, 2009). Based on this representation, the correspondence between model elements and solution elements is established, as shown in Fig. 4. A supply chain corresponds to a solution, which can be divided into several fragments. Each fragment represents a supply chain stage, such as supply, production, and distribution. Each element of a fragment is termed as a cell. It consists of two properties: location and value.

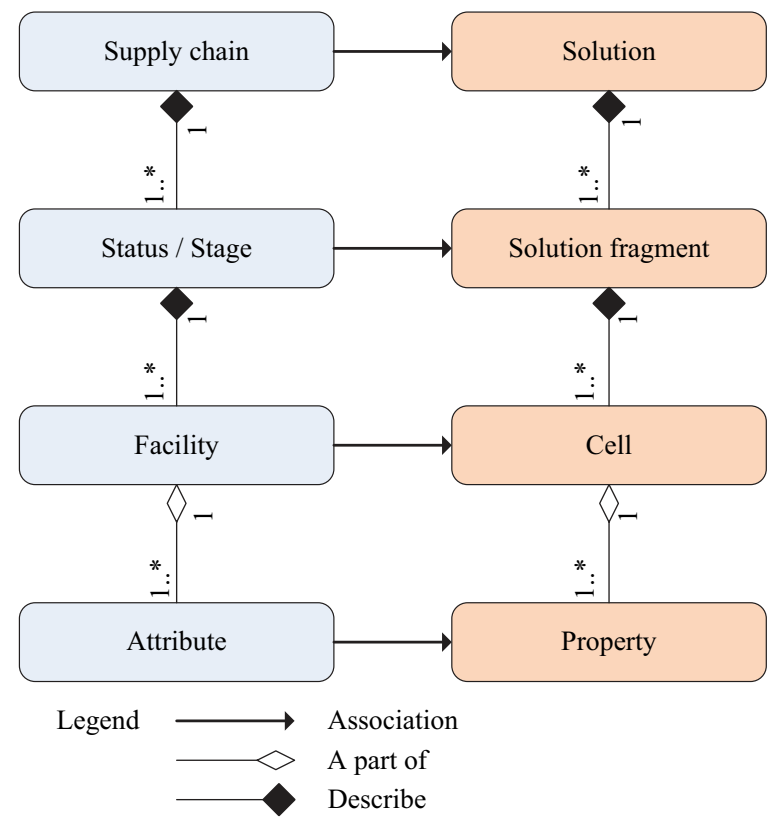

Fig. 4. The correspondence between supply chains and solution elements.

\begin{tabular}{|l|l|l|l|l|l|l|l|l|l|}
\hline \multicolumn{3}{|c|}{ Suppliers } & \multicolumn{3}{c|}{ Plants } & \multicolumn{3}{c|}{ DCs } \\
\hline 1 & 0 & 1 & 0 & 1 & 1 & 0 & 1 & 1 & 1 \\
\hline
\end{tabular}

Fig. 5. The encoding scheme for the first section of a solution.

The location represents the index of the corresponding facility in different fragments; the value describes either the status or the priority of the facility depending on the corresponding fragment.

In a solution, the first section comprises three fragments representing the binary-based encoding mechanism for suppliers, plants, and DCs, respectively. Fig. 5 illustrates the first section, which involves the open status of two suppliers (the first and third supplier), two plants (the first and second plant), and three DCs among four suppliers, three plants and three DCs.

Representing the transportation of products among each pair of facilities in different echelons, the second section contains three fragments, each of which represents a stage. Three fragments correspond to the product transportation from supplier to plant, from plant to DC and from DC to customers, respectively. As an example, the priority-based encoding scheme for the third stage is shown in Fig. 6. In this example, there are three DCs and four customer zones. The location of the element at this stage indicates if it is a DC or a customer zone and the index of the corresponding facility. The value of the element at this stage is the priority of the corresponding facility. The combination of location and priority information guarantees the decoding of the solution scheme. The first and second stages are encoded in a same way. In accordance with the binary-based encoding for the first section and the prioritybased encoding for the second section, we encode the whole solution as shown in Fig. 7.

\begin{tabular}{|c|c|c|c|c|c|c|c|}
\hline Node & DC1 & DC2 & DC3 & CZ1 & CZ2 & CZ3 & CZ4 \\
\hline Priority & 1 & 2 & 7 & 5 & 6 & 4 & 3 \\
\hline
\end{tabular}

Fig. 6. The priority-based encoding scheme for the third stage. 


\begin{tabular}{|c|c|c|c|c|c|c|c|c|c|c|c|c|c|c|c|c|c|c|c|c|c|c|c|c|c|c|c|c|c|}
\hline \multicolumn{4}{|c|}{ Supplier } & \multicolumn{3}{|c|}{ Plant } & \multicolumn{3}{|c|}{$\mathrm{DC}$} & \multicolumn{7}{|c|}{$1^{\text {st }}$ stage } & \multicolumn{6}{|c|}{$2^{\text {nd }}$ stage } & \multicolumn{7}{|c|}{$3^{\text {rd }}$ stage } \\
\hline 1 & 0 & 1 & 0 & 1 & 1 & 0 & 1 & 1 & 1 & 3 & 1 & 7 & 4 & 5 & 6 & 2 & 5 & 1 & 3 & 6 & 4 & 2 & 1 & 2 & 7 & 5 & 6 & 4 & 3 \\
\hline
\end{tabular}

Fig. 7. The encoding scheme for the solution.

The decoding procedure begins from the third stage, through which the actual throughput for each $\mathrm{DC}$ is determined by referring to the open/close status of DCs in the first section of the solution. After that, the second stage is decoded considering the actual throughput of DCs acquired in last step. The first stage is decoded in a same way with the actual throughput of DCs.

\subsection{Constraint handling}

The diverse constraints in the MINLP model originate from a variety of sources, such as demand, production capacity, and BOMs. These constraints are closely related to the set of possible solutions, from which an optimal one is to be found. Therefore, constraint handling suggests itself to be an important issue in solving the proposed model. In this study, we develop a two-step approach to deal with constraints. In the first step, constraints are classified into two types: capacity-related constraints and priority-related constraints. Capacity-related constraints are these constraints that are associated with entities themselves with respect to production capacity, set-up cost, DC throughput, and so on. Priority-related constraints relate to solution from a holistic view, thus modeling BOM constraints, supplier priorities and facility pairwise relationships. In other words, the capacity-related constraints have influence on entities themselves, whilst the priority-related constraints affect the structure of the supply chains.

In the second step, a constraint handling module is designed as a filter functioning at the beginning of the solving process and during the solving process. Initially, constraint rules are stored in a pool. Whenever a new solution is produced, the constraint handling module checks it against the available capacity and priority constraints in the pool. During the solving process, in order to facilitate a diversified evolution, it is necessary to include both feasible and infeasible solutions in the interactive search process. In this study, different penalty factors are assigned to the violations of capacity and priority related constraints at different degrees. Such a mechanism allows both the feasible and infeasible solutions to contribute to the diversified evolution.

\subsection{Phase 1 - Random initialization}

Using the $A B C$ algorithm, in the first phase, a number of solutions are generated randomly as initial solutions. To produce feasible solutions and facilitate the convergent process, in the first section of the solution, the status settings of suppliers, plants, and DCs should consider the demand from customers. For the second section of the solution, the priority in each stage is randomly assigned considering the number of facilities in each stage.

\subsection{Phase 2 - Employed bee phase}

Once the initialization phase is done, employed bees are assigned with initially found food sources. When exploiting food sources, the employed bees also try to search around for better food sources. Such a procedure is implemented through a neighborhood search for each solution in the $A B C$ algorithm. Fig. 8 illustrates how the neighborhood search is conducted. Given the current solution ( $\mathrm{S} 1$ in the figure), another solution ( $\mathrm{S} 2$ in the figure) is randomly chosen from the solution set. Then S1 interacts with S2 stage by stage proportionally. The new generated solution (New in the figure) is taken only if its fitness is over the current solution. This entails the greedy selection. Besides, a local search procedure is conducted for obtaining a new solution after the two available ones interact. In the local search, the value for each cell is regenerated considering its location and range. For instance, if it is located in the first section of the solution, the value could be only adopted as 1 or 0 . Fig. 9 summarizes the local search in this phase.

\subsection{Phase 3 - Onlooker bee phase}

In this phase, the onlooker bees are assigned to explore and exploit the food sources by referring to the information that they have obtained from the employed bees. Their decision making is affected by the richness of food sources. The richer food source certainly could attract more onlooker bees, which facilitates the intensive search in the promising areas. In the ABC algorithm, such a

\begin{tabular}{|c|c|c|c|c|c|c|c|c|c|c|c|c|c|c|c|c|c|c|c|c|c|c|c|c|c|c|c|c|c|c|}
\hline \multirow[b]{2}{*}{$\mathrm{S} 1$} & \multicolumn{4}{|c|}{ Supplier } & \multicolumn{3}{|c|}{ Plant } & \multicolumn{3}{|c|}{ DC } & \multicolumn{7}{|c|}{$1^{\text {st }}$ stage } & \multicolumn{6}{|c|}{$2^{\text {nd }}$ stage } & \multicolumn{7}{|c|}{$3^{\text {rd }}$ stage } \\
\hline & 1 & 0 & 1 & 0 & 1 & 1 & 0 & 1 & 1 & 1 & 3 & 1 & 7 & 4 & 5 & 6 & 2 & 5 & 1 & 3 & 6 & 4 & 2 & 1 & 2 & 7 & 5 & 6 & 4 & 3 \\
\hline & \multicolumn{4}{|c|}{1} & \multicolumn{3}{|c|}{0} & \multicolumn{3}{|c|}{0} & \multicolumn{7}{|c|}{1} & \multicolumn{6}{|c|}{0} & \multicolumn{7}{|c|}{0} \\
\hline S2 & 0 & 1 & 1 & 1 & 1 & 0 & 1 & 0 & 1 & 1 & 5 & 6 & 7 & 4 & 3 & 1 & 2 & 6 & 5 & 2 & 1 & 4 & 3 & 5 & 7 & 6 & 3 & 2 & 1 & 4 \\
\hline New & 0 & 1 & 1 & 1 & 1 & 1 & 0 & 1 & 1 & 1 & 5 & 6 & 7 & 4 & 3 & 1 & 2 & 5 & 1 & 3 & 6 & 4 & 2 & 1 & 2 & 7 & 5 & 6 & 4 & 3 \\
\hline
\end{tabular}

Fig. 8. The neighborhood search.

\begin{tabular}{|c|c|c|c|c|c|c|c|c|c|c|c|c|c|c|c|c|c|c|c|c|c|c|c|c|c|c|c|c|c|c|}
\hline \multirow[b]{2}{*}{$\mathrm{S} 1$} & \multicolumn{4}{|c|}{ Supplier } & \multicolumn{3}{|c|}{ Plant } & \multicolumn{3}{|c|}{$\mathrm{DC}$} & \multicolumn{7}{|c|}{$1^{\text {st }}$ stage } & \multicolumn{6}{|c|}{$2^{\text {nd }}$ stage } & \multicolumn{7}{|c|}{$3^{\text {rd }}$} \\
\hline & 1 & 0 & 1 & 0 & 1 & 1 & 0 & 1 & 1 & 1 & 3 & 1 & 7 & 4 & 5 & 6 & 2 & 5 & 1 & 3 & 6 & 4 & 2 & 1 & 2 & 7 & 5 & 6 & 4 & 3 \\
\hline $\mathrm{S} 2$ & 0 & 0 & 1 & 0 & 1 & 1 & 0 & 1 & 0 & 1 & 3 & 5 & 7 & 4 & 1 & 6 & 2 & 5 & 1 & 3 & 6 & 4 & 2 & 3 & 2 & 7 & 5 & 6 & 4 & 1 \\
\hline
\end{tabular}

Fig. 9. The local search. 


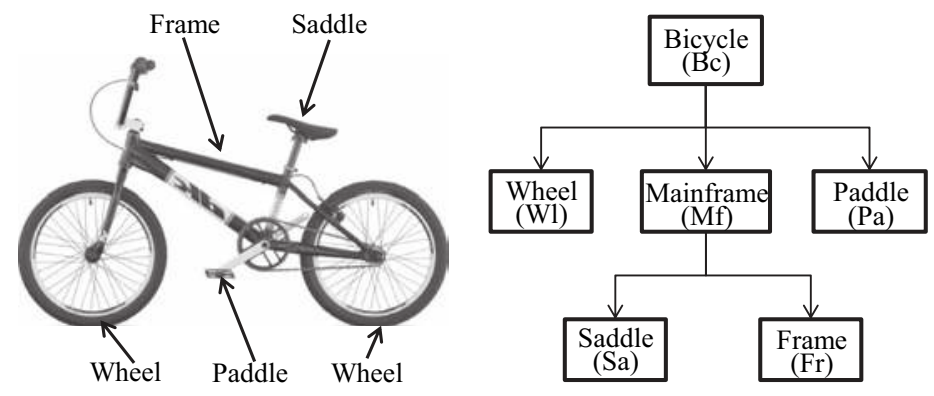

Fig. 10. The BOM structure of bicycles.

Table 1

The characteristics of the four scenarios.

\begin{tabular}{lllll}
\hline Scenario \# & $\begin{array}{l}\text { Number of } \\
\text { suppliers }\end{array}$ & $\begin{array}{l}\text { Number } \\
\text { of plants }\end{array}$ & $\begin{array}{l}\text { Number } \\
\text { of DCs }\end{array}$ & $\begin{array}{l}\text { Number } \\
\text { of CZs }\end{array}$ \\
\hline 1 & 4 & 3 & 3 & 4 \\
2 & 8 & 5 & 6 & 8 \\
3 & 10 & 8 & 10 & 9 \\
4 & 15 & 10 & 12 & 10 \\
\hline
\end{tabular}

procedure is implemented using a roulette wheel selection mechanism considering the fitness of solutions. Once a decision is made, the onlooker bees become employed bees and conduct neighborhood search for better food sources.

\subsection{Phase 4 - Scout bee phase}

An abandonment criterion is necessary for escaping the local optima and for increasing the diversified search in the solutions space. In other words, if one solution can not be improved after a certain number of iterations, it is replaced by another solution, which is randomly found by scout bees.

\section{An industrial example}

Compared with other typical products (e.g., automobiles), bicycles are less complex, and do not involve too many product components. Moreover, the degree of product complexity and the number of product components suggest bicycles to be suitable industrial products for case studies (Kotha, 1996; Magill, 1996; Ong, Lin, \& Nee, 2006). Thus, in this study, we use the bicycle to demonstrate the feasibility and potential of the proposed MINLP supply chain design model and the ABC-based solving approach. Fig. 10 shows a bicycle, the main components, and the general product structure. In general, a bicycle has three immediate child components, including a wheel $(\mathrm{Wl})$ representing both the front and back wheels, mainframe (Mf), and paddle (Pa) denoting the paddle system including the chain. The mainframe is further formed by a saddle (Sa) and a frame (Fr). (Note, As Wl represents both the front and back wheels, each bicycle needs one Wl. In this regard, the unit per of each component in the figure is 1 , thus being omitted.)

To produce and deliver bicycles, four layers are involved in the supply chain, including component suppliers, main plants, DCs, and CZs. While mainframes and bicycles are produced in the main plants, the other components are produced by suppliers. To validate the proposed model and ABC-based solution approach, we consider four scenarios, as shown in Table 1 . These scenarios differ from each other in the supply chain structure with respect to the number of supply chain facilities. Along with the increase of the number of facilities in each layer, the problem complexity and size of these scenarios increases exponentially. To demonstrate the problem solving performance of the $\mathrm{ABC}$-based approach by means of comparison, we adopt the GA and LINDO to solve the four scenarios. Both the ABC-based and GA-based approaches are implemented using Java.

In the first scenario, there are four suppliers (one for $\mathrm{Wl}$, one for Pa, and two alternatives for Sa and Fr), three plants (for both Mf and $\mathrm{Bc}$ ), three DCs and four CZs. The design capacities, production capacities, set-up costs, and demand of these facilities are given in Table 2. As shown in the table, customer demands (in $\mathrm{K}$ units) from the four CZs are 580, 430, 460, and 350, respectively. According to the definition in the proposed model, a supplier design

Table 2

Design/production capacities, set-up costs, and demand in scenario 1.

\begin{tabular}{lllll}
\hline Supply chain facility \# & $\# 1$ & $\# 2$ & $\# 3$ & $\# 4$ \\
\hline Customer demand & 580 & 430 & 460 & 350 \\
Supplier design capacity & Wl/0.9 & $\mathrm{Sa} / 0.6 ; \mathrm{Fr} / 0.85$ & $\mathrm{Sa} / 0.8 ; \mathrm{Fr} / 0.9$ & $\mathrm{~Pa} / 0.75$ \\
Supplier production capacity & 1820 & 1900,1900 & 2000,2000 & 2100 \\
Plant design capacity & $M f / 0.88 ; \mathrm{Bc} / 0.95$ & $\mathrm{Mf} / 0.95 ; \mathrm{Bc} / 0.85$ & $\mathrm{Mf} / 0.90 ; \mathrm{Bc} / 1$ & \\
Plant production capacity & 900 & 1150 & 1380 \\
DC maximum throughput & 1250 & 1080 & 1050 \\
Plant set-up cost & 2500 & 2200 & 2800 \\
DC set-up cost & 1200 & 1800 & 1000 & \\
\hline
\end{tabular}

Table 3

Transportation costs between two facilities in scenario 1 .

\begin{tabular}{lrlllllllllll}
\hline Supplier/Plant & $\# 1$ & \# 2 & \# 3 & Plant/DC & $\# 1$ & $\# 2$ & \# 3 & DC/CZ & $\# 1$ & $\# 2$ & $\# 3$ & $\# 4$ \\
\hline \# 1 & 18 & 15 & 20 & $\# 1$ & 8 & 6 & 9 & $\# 1$ & 25 & 22 & 18 & 20 \\
$\# 2$ & 12 & 14 & 16 & $\# 2$ & 9 & 12 & 10 & $\# 2$ & 17 & 21 & 24 & 22 \\
$\# 3$ & 11 & 17 & 15 & $\# 3$ & 7 & 10 & 13 & $\# 3$ & 15 & 19 & 23 & 20 \\
$\# 4$ & 8 & 13 & 11 & & & & & & & & & \\
\hline
\end{tabular}




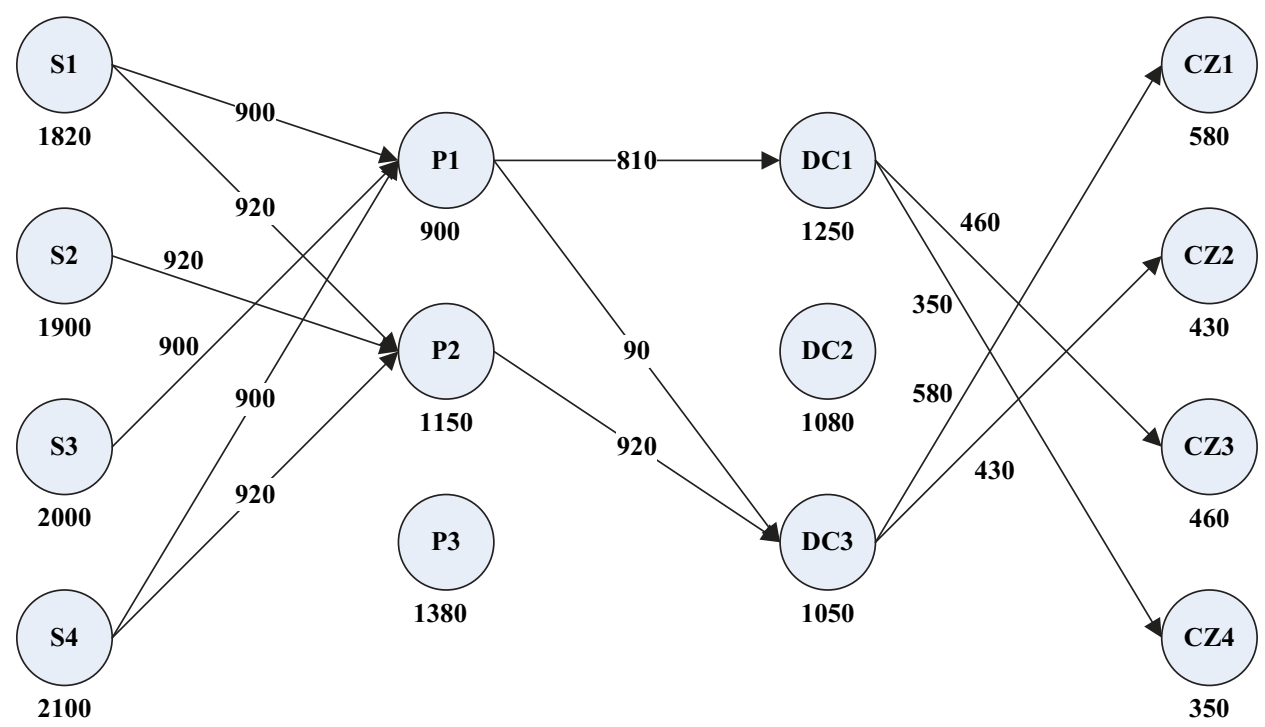

Fig. 11. The supply chain obtained using LINDO.

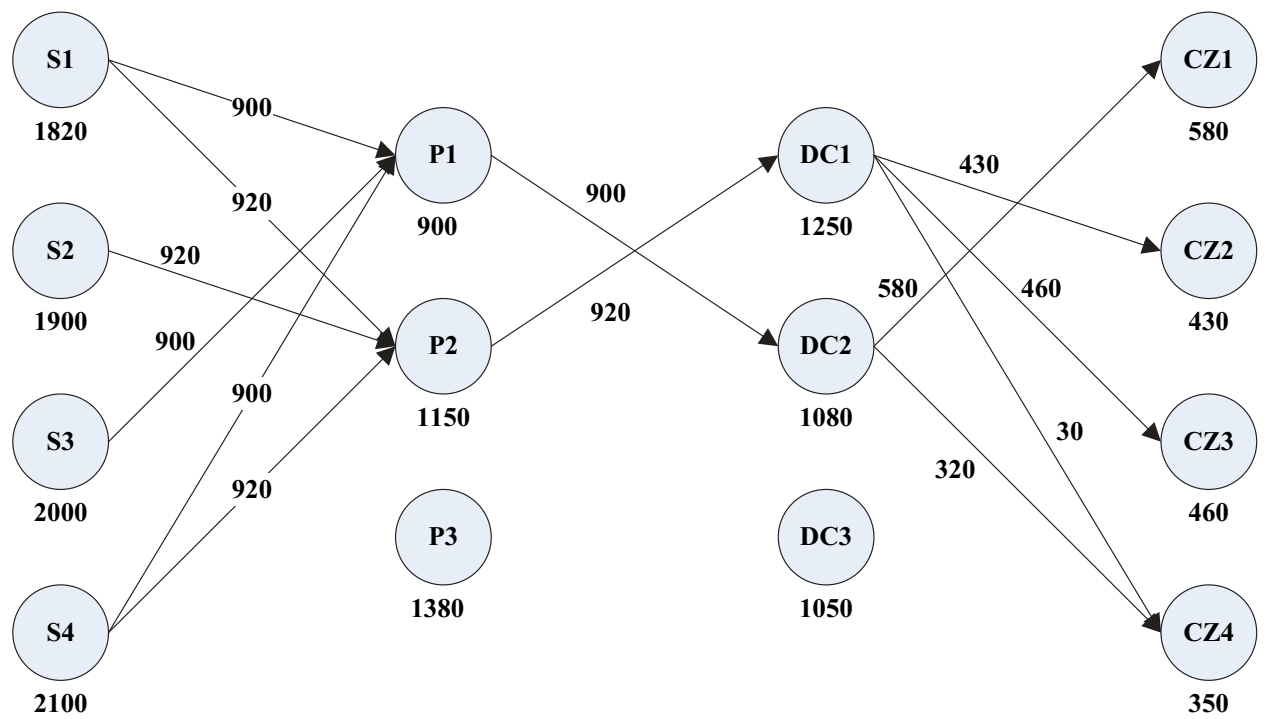

Fig. 12. The supply chain obtained using the $A B C$-based approach.

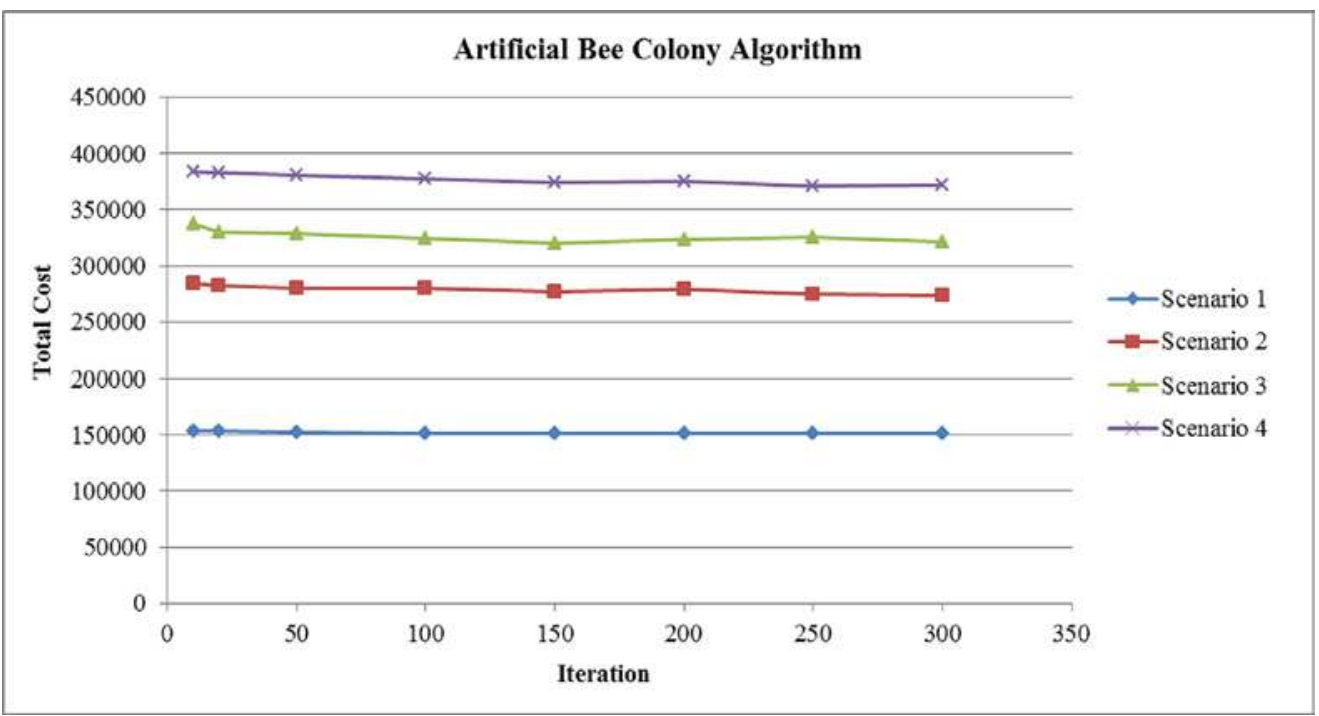

Fig. 13. The performance of the $A B C$-based approach. 
capacity indicates the probability that a supplier can undertake the task to design a component. For example, Sa/0.6 and Fr/0.85 represent that the probabilities for Supplier 2 to design $\mathrm{Sa}$ and $\mathrm{Fr}$ are 0.6 and 0.85 , respectively. Similarly, the plant design capacities indicate the probabilities of the three plants to design mainframes and bicycles. Supplier/plant production capacities (in K units) represent the production capacities of the four suppliers and the three plants. Plant/DC set-up costs are the set-up costs (in K dollars) for plants/DCs to produce/deliver bicycles. DC maximum throughputs represent the maximum number (in $\mathrm{K}$ units) of bicycles that DCs can handle. In regard to supplier priority determination, supplier design capacities are used as reference. Higher priorities are assigned to suppliers with higher design capacities and lower priorities are assigned to suppliers with lower design capacities for the same components. For example, since Supplier \#3 has higher design capacities for both Sa and Fr than Supplier \#2, the priorities assigned to Supplier \#3 for both components are higher than Supplier \#2. In accordance with these priorities, Supplier \#2 or Supplier \#3 or both will be included in the possible supply chains that can be obtained. Since Supplier \#1 and Supplier \#4 are the only suppliers to produce $\mathrm{Wl}$ and $\mathrm{Pa}$, respectively, they will be included in all possible supply chains.

The transportation costs (in K dollars) between two facilities at two adjacent layers are given in Table 3.

This scenario is first solved using LINDO. The optimal solution with respect to the facilities and their production/delivery quantities is given in Fig. 11. As shown, the supply chain involves all four suppliers, 2 plants (plants 1 and 2), 2 DCs (DCs 1 and 3), and all four CZs. In this solution, all customer requirements are met. Without exceeding its production capacity: $1820 \mathrm{~K}$, Supplier \#1 produces $900 \mathrm{~K}$ and $920 \mathrm{~K}$ wheels for plants 1 and 2, respectively. Supplier \#2 delivers both $920 \mathrm{~K}$ saddles and $920 \mathrm{~K}$ frames to Plant \#2; Supplier \#3 transports $900 \mathrm{~K}$ saddles and $900 \mathrm{~K}$ frames to Plant \#1. Supplier \#4 produces $900 \mathrm{~K}$ and $920 \mathrm{~K}$ paddles for plants 1 and 2, respectively. While Supplier \#1 fully utilizes its production capacity, suppliers 2,3 , and 4 do not fully utilize their capacities. Plants 1 and 2 deliver $900 \mathrm{~K}$ and $920 \mathrm{~K}$ bicycles to the two DCs. There is no production capacity left at Plant \#1, whilst there is capacity left (i.e., $230 \mathrm{~K}$ ) at Plant \#2. DCs 1 and 3 deliver $460 \mathrm{~K}, 350 \mathrm{~K}, 580 \mathrm{~K}$, and $430 \mathrm{~K}$ bicycles to the four CZs, respectively. The corresponding supply chain cost is 150260 unit of cost.

In using the proposed $A B C$-based approach to solve this scenario, the size of the colony is set as 80 including 40 employed bees and 40 onlooker bees. 40 initial solutions are generated during the process. Each operation is executed 10 times and the average value is used to denote the overall cost. The supply chain obtained consists of all four suppliers, two plants (plants 1 and 2), two DCs (DCs 1 and 2), and all four CZs, as shown in Fig. 12. Fig. 12 also shows the quantities shipped between two of these facilities. The cost for this supply chain is 151340 unit of cost. For this scenario, the GA-based approach acquires the same result as the ABCbased approach. Although the supply chain cost: 151340 obtained using the $A B C$-based approach is slightly higher than that obtained using LINDO: 150260 , the increase percentage is only $0.7 \%$. In this regard, the $A B C$-based solution approach is effective in finding near optimal solutions. (Note: As the ABC algorithm is meta-heuristics, the $\mathrm{ABC}$-based solution approach is not able to find optimal solutions.)

Fig. 13 shows the performance (with respect to the number of iterations and costs) of the ABC-based approach in solving all scenarios. The convergent curves fluctuate due partially to the newly introduced parameters, variables and constraints in scenarios 2, 3, and 4 and partially to the discreteness of decision variables. The ABC-based approach can find a satisfactory solution within 200 generations for Scenario 1. However, due to the increased problem
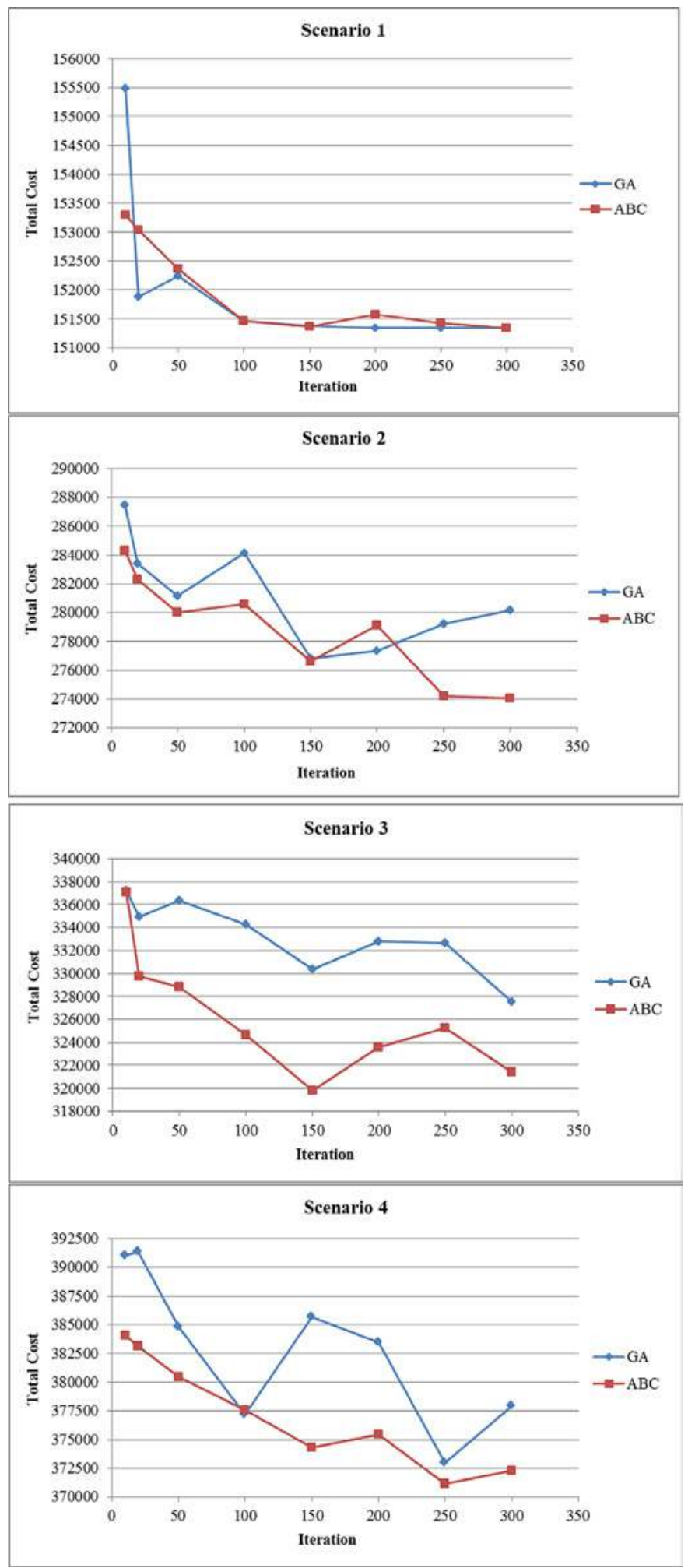

Fig. 14. The comparison between the ABC-based and GA-based approaches.

complexity, it needs more generations to achieve a satisfactory convergence for Scenario 2, 3 and 4.

To compare the performance of LINDO, ABC-based and GAbased approaches in solving these four scenarios, the results in terms of the supply chain cost obtained are provided in Table 4. With the increase of the number of supply chain facilities, parameters, decision variables, and constraints in each scenario increase 
Table 4

Performance comparison of LINDO, ABC-based and GA-based approaches.

\begin{tabular}{llll}
\hline Scenario & $\begin{array}{l}\text { Lindo } \\
\text { Cost obtained }\end{array}$ & $\begin{array}{l}\text { ABC-based approach } \\
\text { Cost obtained }\end{array}$ & $\begin{array}{l}\text { GA-based approach } \\
\text { Cost obtained }\end{array}$ \\
\hline 1 & 150260 & 151340 & 151340 \\
2 & 246120 & 273440 & 273810 \\
3 & 1 & 321450 & 327100 \\
4 & 1 & 371070 & 374710 \\
\hline
\end{tabular}

substantially. This, in turn, increases problem size and complexity of the scenarios. LINDO cannot solve scenarios 3 and 4, which are of large problem size and high problem complexity. The ABCbased and GA-based approaches can solve all these scenarios with slightly increased solving time.

To further demonstrate the efficiency of the $A B C$ algorithm, we compare the performance of the ABC-based and GA-based approaches in solving each scenario (in Fig. 14). As shown, the ABCbased approach exceeds the GA-based approach with better results and less fluctuation. There are several reasons for this. First, the ABC-based approach consists of other three phases besides the initialization phase and each phase can be treated as an individual process. Second, the greedy selection mechanism in the ABC-based approach facilitates the convergence of the solutions.

To conclude, the ABC-based approach can solve all the scenarios with good performance. For small size problems, LINDO may perform the best as it uses exact mechanism to search the whole solution space. However, for large scale problems, LINDO is not able to find the optimal solutions because of the large number of parameters, decision variables, and constraints. On the contrary, the $\mathrm{ABC}$-based approach is more efficient in solving problems with many parameters, decision variables, and constraints. In addition, the unique structure and the inner mechanisms of the ABC-based approach guarantee the better performance against the GA-based approach.

\section{Conclusions}

This study developed an MINLP model and ABC-based solution approach for designing supply chains. In the broad sense, the model and solution approach form an expert system providing practical decision making support in supply chain design. First, unlike many available supply chain design models, the MINLP model takes into account three major supply chain stages: procurement, production, and distribution and their interactions and involves BOM-related constraints. Thanks to these unique model features, the expert system as a whole can help design realistic supply chains. Second, as today's companies develop product families, instead of single products, involving multiple products in the MINLP model also facilitates the design of practical supply chains. Third, thanks to the inclusion of constraints related to facility pairwise relationship and supplier priority, the expert system also contributes to designing practical supply chains. Last, as the ABC-based solution approach performs better than the commercial solver LINDO and GA-based approach, the system as a whole can help design more complex supply chains and reduce supply chain costs.

While this study has the above contributions and significance, like most of the reported studies, it has certain limitations. First, as an initial study investigating supply chain design by integrating all the three important stages, the MINLP model considers one period in the planning horizon, thus being deterministic by nature. Consequently, the model cannot deal with environmental dynamics especially with respect to demand variations, which can be observed in many industries nowadays. In this regard, the future efforts might be directed to develop a multi-period model which takes into account stochastic demand. Second, in formulat- ing the MINLP model, we set cost as a single criterion in the objective function. As in some situations, companies might pursue several performance measures at the same time, e.g., cost, profit. In this regard, the MINLP model is not applicable in the situations where companies consider other performance measures besides cost. Thus, the second potential avenue for future research is to develop a comprehensive model considering multi-objective, multi-period, and all the three stages. Third, there is also potential future research regarding the solution approach. Efforts might be made to integrate the ABC-based approach with other programming methods to further improve its performance. Fourth, an expert system in the narrow sense (i.e., a computer system), where the above comprehensive supply chain design model and a solution method are embedded as system components, might be developed. The system should be flexible enough such that it can be used to design supply chains in different situations.

\section{Appendix A. Notation used in the model}

\section{Appendix A.1. Indices}

$i \in I \quad$ A set of products offered to customers;

$j \in J \quad$ A set of candidate suppliers;

$k \in K \quad$ A set of potential plants;

$l \in L \quad$ A set of DCs;

$m \in M \quad$ A set of CZs; and

$r \in R \quad$ A set of components provided by suppliers.

\section{Appendix A.2. Input parameters}

$f_{r j}^{S} \quad$ Set-up cost for supplier $j$ to produce component $r$;

$f_{i k}^{P} \quad$ Set-up cost for plant $k$ to produce product $i$;

$f_{i l}^{D} \quad$ Set-up cost for DC $l$ to deliver product $i$;

$\delta_{i k} \quad$ Capacity of plant $k$ to design product $i$;

$\delta_{r j}^{i k} \quad$ Capacity of supplier $j$ to design component $r$;

$c_{r j k}^{S P} \quad$ Unit transportation cost from supplier $j$ to plant $\mathrm{k}$ for component $r$ (\$/unit);

$c_{i k l}^{P D} \quad$ Unit transportation cost from plant $k$ to DC $l$ for product $i$ (\$/unit);

$c_{i l m}^{D Z} \quad$ Unit transportation cost from $\mathrm{DC} l$ to $\mathrm{CZ}$ m for product $i$ (\$/unit);

$p_{r j}^{S} \quad$ Unit purchasing cost of component $r$ from supplier $j$ (\$/unit);

$p_{i k}^{P} \quad$ Unit production cost of product $i$ at plant $k$ (\$/unit);

$p_{i l}^{D} \quad$ Unit cost of throughput (i.e., cost of handling and inventory) of product $i$ at DC $l$ (\$/unit);

$\Phi_{r j}^{S} \quad$ Maximum production capacity of supplier $j$ for component $r$ (units/period);

$\Phi_{i k}^{P} \quad$ Maximum production capacity of plant $k$ for product $i$ (units/period);

$\Phi_{k}^{P} \quad$ Total production capacity of plant $k$ (units/period);

$\Phi_{l m}^{D} \quad$ Maximum throughput of DC $l$ (units/period);

$\lambda_{r i} \quad$ Quantity of component $r$ used in one unit of product $i$ (unit);

$\alpha_{j} \quad$ Service level of supplier $j$;

$\beta_{k} \quad$ Service level of plant $k$;

$\gamma_{l} \quad$ Service level of DC $l$;

$\eta_{r j} \quad$ Cost coefficient for supplier $j$ to design component $r$;

$\eta_{i k} \quad$ Cost coefficient for plant $k$ to design product $i$; and

$D_{i m} \quad$ Average demand for product $i$ at $C Z m$ (units/period).

Appendix A.3. Decision variables

$X_{i k} \quad$ Volume of product $i$ produced at plant $k$ (units/period); 


\begin{tabular}{|c|c|}
\hline$Y_{i l}$ & olume of product $i$ received at DC $l$ (units/period); \\
\hline & $\begin{array}{l}\text { olume of component } r \text { provided by supplier } j \\
\text { units/period); }\end{array}$ \\
\hline$Q_{i k l}$ & $\begin{array}{l}\text { Volume of product } i \text { transferred from plant } k \text { to DC } \\
l \text { (units/period); }\end{array}$ \\
\hline$r j k$ & $\begin{array}{l}\text { Volume of component } r \text { transferred from supplier } j \\
\text { to plant } k \text { (units/period); }\end{array}$ \\
\hline ilm & $\begin{array}{l}\text { roduct } i \text { transferred from DC } l \text { to } C Z m \\
\text { ); }\end{array}$ \\
\hline$U_{r j}=\{0,1\}$ & $\begin{array}{l}R \text { A binary variable indicating whether } \\
\text { open for component } r \text {; }\end{array}$ \\
\hline$V_{i k}=\{0,1\}$ & $\begin{array}{l}\text { riable indicating whether } \\
i \text {; }\end{array}$ \\
\hline$W_{i l}=\{0,1\}$ & $\begin{array}{l}\text { I A binary variable indicating whether } \\
\text { for product } i \text {; }\end{array}$ \\
\hline$T_{i l m}=\{0,1\}$ & $\in M$ A binary variable indicating \\
\hline$A_{i}=\{0,1\}$ & $\begin{array}{l}\text { for } i \in I \text {, A binary variable indicating whether prod- } \\
\text { uct } i \text { is produced; and }\end{array}$ \\
\hline$r i=\{0,1\}$ & $\begin{array}{l}\text { for } r \in R, i \in I \text {, A binary variable indicating wheth } \\
\text { component } r \text { is used in product } i \text {. }\end{array}$ \\
\hline
\end{tabular}

\section{Supplementary Materials}

Supplementary material associated with this article can be found, in the online version, at doi:10.1016/j.eswa.2015.12.035.

\section{References}

Altiparmak, F., Gen, M., Lin, L., \& Karaoglan, I. (2009). A steady-state genetic algorithm for multi-product supply chain network design. Computers $\mathcal{E}$ Industrial Engineering, 56, 521-537.

Anussornnitisarn, P., Nof, S. Y., \& Etzion, O. (2005). Decentralized control of cooperative and autonomous agents for solving the distributed resource allocation problem. International Journal of Production Economics, 98, 114-128.

Arntzen, B., Brown, G., Harrison, T., \& Trafton, L. L. (1995). Global supply chain management at Ditital Equipment corporation. Interfaces, 25, 69-93.

Bonabeau, E., Dorigo, M., \& Theraulaz, G. (1999). Swarm intelligence: From natural to artificial systems. New York: Oxford University Press.

Chung, S. H., Lau, H. C. W., Choy, K. L., Ho, G. T. S., \& Tse, Y. K. (2010). Application of genetic approach for advanced planning in multi-factory environment. International Journal of Production Economics, 127, 300-308.

Cohen, M. A., \& Lee, H. L. (1989). Resource deployment analysis of global manufacturing and distribution networks. Journal of Manufacturing and Operations Management, 2, 81-104.

Das, K. (2011). Integrating effective flexibility measures into a strategic supply chain planning model. European Journal of Operational Research, 211, 170-183.

Dominguez, H., \& Lashkari, R. S. (2004). Model for integrating the supply chain of an appliance company: A value of information approach. International Journal of Production Research, 42, 2113-2140.

Gebennini, E., Gamberini, R., \& Manzini, R. (2009). An integrated productiondistribution model for the dynamic location and allocation problem with safety stock optimization. International Journal of Production Economics, 122, 286-304.

Huang, G. Q. Zhang, X. Y., \& Liang, L. (2005). Towards integrated optimal configuration of platform products, manufacturing processes, and supply chains. Journal of Operations Management, 23, 267-290.
Horng, M. H. (2011). Multilevel thresholding selection based on the artificial bee colony algorithm for image segmentation. Expert Systems with Applications, 38, 13785-13791.

Ivanov, D. (2010). An adaptive framework for aligning (re)planning decisions on supply chain strategy, design, tactics, and operations. International Journal of Production Research, 48, 3999-4017.

Karaboga, D. (2005). An idea based on honey bee swarm for numerical optimization, Technical report-tr06. Computer Engineering Department, Engineering Faculty, Erciyes university.

Karaboga, D., Gorkemli, B., Ozturk, C., \& Karaboga, N. (2014). A comprehensive survey: artificial bee colony $(\mathrm{ABC})$ algorithm and applications. Artificial Intelligence Review, 42, 21-57.

Kim, B., \& Kim, J. (2008). Values of the balanced decision making between supply chain partners. International Transactions in Operational Research, 15, 623-649.

Kotha, S. (1996). From mass production to mass customization: The case of the National Industrial Bicycle Company of Japan. European Management Journal, 14, $442-450$.

Li, H., Hendry, L., \& Teunter, R. (2009). A strategic capacity allocation model for a complex supply chain: formulation and solution approach comparison. International Journal of Production Economics, 121, 505-518.

Magill, J. R. (1996). Customizing bicycles through the integration of best practices. IEE Colloquium Digest, 181, 1-6.

Melo, M. T., Nickel, S., \& Saldanha-da-Gama, F. (2005). Dynamic multi-commodity capacitated facility location: a mathematical modeling framework for strategic supply chain planning. Computers E Operations Research, 33, 181-208.

Melo, M. T., Nickel, S., \& Saldanha-da-Gama, F. (2009). Facility location and supply chain management: a review. European Journal of Operational Research, 196, 401412 .

Moon, C., Seo, Y., Yun, Y., \& Gen, M. (2006). Adaptive genetic algorithm for advanced planning in manufacturing supply chain. Journal of Intelligent Manufacturing, 17, 509-522.

Ong, S. K., Lin, Q., \& Nee, A. Y. C. (2006). Web-based configuration design system for product customization. International Journal of Production Research, 44, 351382 .

Osman, H., \& Demirli, K. (2010). A bilinear goal programming model and a modified Benders decomposition algorithm for supply chain reconfiguration and supplier selection. International Journal of Production Economics, 124, 97-105.

Sabri, E. G., \& Beamon, B. M. (2000). A multi-objective approach to simultaneous strategic and operational planning in supply chain design. Omega, 28, 581-598.

Safaei, A. S., Moattar Husseini, S. M., \& Farahani, R. Z. (2010). Integrated multi-site production-distribution planning in supply chain by hybrid modeling. International Journal of Production Research, 48, 4043-4069.

Simchi-Levi, D., Kaminsky, P., \& Simchi-Levi, E. (2004). Managing the supply chain: the definitive guide for the business professional. New York: McGraw-Hill.

Simchi-Levi, D., Kaminsky, P., \& Simchi-Levi, E. (2009). Designing and managing the supply chain: concepts strategies and case studies (3rd ed.). New York: McGrawHill.

Thanh, P. N., Bostel, N., \& Peton, O. (2008). A dynamic model for facility location in the design of complex supply chains. International Journal of Production Economics, 113, 678-693.

Van der Vaart, T., \& van Donk, D. P. (2008). A critical review of survey-based research in supply chain integration. International Journal of Production Economics, $111,42-55$.

Vidal, C. J., \& Goetschalckx, M. (1997). Strategic production-distribution models: a critical review with emphasis on global supply chain models. European Journal of Operational Research, 98, 1-18.

Wang, J., \& Lin, H. Y. (2006). A fuzzy hybrid decision-aid model for selecting partners in the design chain. International Journal of Production Research, 44, $2047-$ 2069.

Yan, H., Yu, Z., \& Cheng, E. (2003). A strategic model for supply chain design with logical constraints: formulation and solution. Computers \& Operations Research, 30, 2135-2155.

Zhang, L., You, X., Jiao, J., \& Helo, P. (2009). Supply chain configuration with coordinated product, process and logistics decision: an approach based on Petri nets. International Journal of Production Research, 47, 6681-6706. 